\title{
Design and Preliminary Testing of a High Performance Antiproton Trap (HiPAT)
}

\author{
James Martin ${ }^{1}$, Raymond Lewis ${ }^{2}$, Kevin Kramer $^{3}$, Kirby Meyer ${ }^{3}$ and Gerald Smith ${ }^{4}$ \\ ${ }^{1}$ NASA MSFC, TD40, Huntsville, Alabama, 35812 \\ ${ }^{2} R$. Lewis Company (MSFC, TD40), Huntsville, Alabama, 35812 \\ ${ }^{3}$ Penn State University, 19 Research East, University Park, PA 16801 \\ ${ }^{4}$ Synergistic Technologies Inc, 127 East Gate Dr. Suite 203, Los Alamos, NM 87544 \\ Jim.Martin@msfc.nasa.gov/(256) 544-6054 / Fax (256) 544-5926
}

\begin{abstract}
Antimatter represents the pinnacle of energy density, offering the potential to enhance current fusion/fission concepts enabling various classes of deep space missions. Current production rates are sufficient to support proof-ofconcept evaluation of many key technologies associated with antimatter-derived propulsion. Storage has been identified as a key enabling technology for all antimatter-related operations, and as such is the current focus of this NASA-MSFC effort to design and fabricate a portable device capable of holding up to $10^{12}$ particles. Hardware has been assembled and initial tests are underway to evaluate the trap behavior using electron gun generated, positive hydrogen ions. Ions have been stored for tens of minutes, limited by observed interaction with background gas. Additionally, radio frequency manipulation is being tested to increase lifetime by stabilizing the stored particles, potentially reducing their interaction with background gas, easing requirements on ultimate trap vacuum and precision mechanical alignment.
\end{abstract}

\section{INTRODUCTION}

The proton antiproton annihilation process packs the highest energy density of any known reaction in modern physics. This value at $10^{8} \mathrm{MJ} / \mathrm{g}$ is 10 orders of magnitude above that of current chemical systems, 1000 times that of fission based systems and 100 times that of fusion systems. To put it in terms of current NASA flight systems for perspective, the approximately $750,000 \mathrm{~kg}$ of fuel and oxidizer onboard the space shuttle external tank has the same energy equivalence as $42 \mathrm{mg}$ of antiprotons (roughly $0.6 \mathrm{cc}$ 's in the form of antihydrogen). This potential has clearly provided the impetus for research focused on the use of antimatter as a method of powering a new breed of propulsion systems for deep space missions. Past studies (Vulpetti, 1984; Morgan, 1982; Rider 1997) have addressed systems ranging from low Isp configurations, deriving thrust by heating an intermediate solid core (similar to nuclear thermal reactors), to those concepts with incredibly high Isp, which directly exhaust the annihilation products (usually referred to as beam core). The shortfall of these concepts is the requirement for incredible amounts of antimatter (kg to tons) since they derive all their energy from the annihilation process. However, several propulsion concepts have been studied that require far fewer antiprotons (micrograms to milligrams) (Gaidos, 1998; Kammash, 1992; Perkins, 1999). These systems derive their energy through a combination of fission/fusion reactions and require only moderate numbers of antiprotons to serve as an initiator or catalyst but still provide moderate Isp's ranging in the tens of thousands of seconds. An attractive feature of these systems if they prove fruitful, is that fusion driver mass is replaced by the energy stored within the antiprotons. The use of these hybrid systems may offer a near term approach to addressing deep space missions utilizing the incredible potential of antimatter combined with fusion energy to enable deep space missions which are impractical (both in time and net material) by any other method (Frisbee, 1998; Borowski, 1987).

To develop any antimatter derived space propulsion system or basic antimatter economy, three key technology areas must be addressed, which include: production, storage and utilization. Currently, within the United States, antiprotons are produced at two Department of Energy facilities: the Fermi and Brookhaven National laboratories. Studies have been performed regarding the current levels of production, efficiencies, and potentials for increase in the near future (Schmidt, 2000; Augenstein, 1988). Currently these sites have a combined production capability of approximately two nanograms annually, quantities more than sufficient for proof of principle evaluations of the remaining two technology areas. Storage of antiprotons has successfully been demonstrated as reported in past 
experiments. These experiments have involved studying as few as two antiprotons for several months (Gabrielse, 1990), up to the storage of almost a million for nearly an hour (Holzscheiter, 1996). Portability has also been examined (Howe, 1988). Basic storage is viewed as a key enabling technology and is currently the focus of the NASA MSFC Propulsion Research Center antimatter activity. To this end, a hardware based test program termed the High Performance Antiproton Trap (HiPAT) has been established. This project is focused on developing a second generation portable trap system building on experience gained from a previous NASA JPL and Penn State University collaboration which resulted in the first generation portable trap referred to as Mark I (Smith, 1997). Once basic portable storage has been successfully demonstrated with antiprotons, utilization experiments could begin in earnest. This activity would initially involve the subscale testing of catalyzed or initiated hybrid propulsion techniques as previously mentioned. Additionally, the availability of trapped low energy antiprotons can be used to investigate high density storage (such as the production of anti-hydrogen) which would be required as the next step in storage technology before it could be utilized for an actual propulsion concept.

\section{TRAP DESIGN REQUIREMENTS}

The HiPAT, a Penning trap that incorporates both magnetic and electric fields to hold charged particles, was designed with a goal of holding $10^{12}$ antiprotons $(\bar{p})$ for up to 18 days. These requirements are believed sufficient from both a quantity and transport perspective to enable proof of concept testing of prospective propulsion concepts. The trap size and inner volume was constrained by the desire for portability and ultimately by project cost. Based on these geometric and performance data the magnetic field, electric field and vacuum requirements could be determined. Figure 1 illustrates the general layout of the electrode structure.

\section{Overview}

Mechanisms that limit the containment time of a cloud in the trap include viscous drag and "chemical" interactions in background gas, and unstable perturbations due to mechanical misalignments. Theoretical models are sufficiently well developed to treat the viscous drag aspect, defining acceptable background gas density and gas composition parameters. However models of the "chemistry", including charge-exchange with ions and $\bar{p}$-atom formation with antiprotons, are less well understood. The effects of alignment perturbations are also obscure, so that only rough guidelines are available for defining trap stability. Fortunately several tools are available for improving the containment time: r.f. manipulation techniques, elaborate and expensive isolation techniques to obtain picotorr level vacuum, and precision alignment mechanisms. The r.f. manipulation is needed regardless as a method to drive particles into the highly-organized motion required for high-density storage. The r.f. also provides stability against viscous drag, misalignment perturbations, and even chemistry, reducing the demands on ultimate vacuum and precision alignment technology.

\section{Requirements for High Density Storage}

The magnetic requirement, providing radial confinement, is set by the necessity to achieve the highest possible ion density. This condition is obtained when the ions in a cloud rotate about the symmetry axis with a common rotation frequency $\omega_{\mathrm{r}}$, with little random (thermal) motion. The density $\mathrm{n}$, cloud rotation and cyclotron frequency are related (Bollinger, 1993) through the plasma frequency,

$$
\omega_{\mathrm{p}}^{2}\left(=q^{2} n / \varepsilon_{0} m\right)=2 \omega_{\mathrm{r}}\left(\omega_{\mathrm{c}}-\omega_{\mathrm{r}}\right),
$$

where $\omega_{c}=q B / m$ is the cyclotron frequency and $q$ is the ion charge. The maximum ion density is achieved with a rotation frequency equal to half the cyclotron frequency. The maximum density, termed the Brillouin (Brillouin, 1945) limit, can be expressed in the form

$$
n=B^{2} /\left(2 \mu_{0} m c^{2}\right)
$$

where $m$ is the mass of a particle (ion, electron, $\bar{p}$, etc.) in the cloud. This relationship between applied field and particle density is illustrated in Figure 2. 
While the magnetic field confines the cloud in the radial direction, axial confinement is achieved by creating an electrostatic potential well using electrodes as shown in Figure 1. At the highest density, particles in the cloud are distributed so that their electric fields cancel the axial component of fields produced by the electrodes. For a spheroidal cloud with diameter $2 r_{0}$ and axial extent $2 z_{0}$, and uniform density $n$, the potential is given by (Bollinger, 1993)

$$
q V(r, z)=-q^{2} n / 6 \varepsilon_{0}\left(a r^{2}+b z^{2}\right)
$$

where $b=3 Q_{1}^{0}\left(\alpha /\left(\alpha^{2}-1\right)^{1 / 2}\right) /\left(\alpha^{2}-1\right)$, with $\alpha=z_{0} / r_{0}$, and $a=(3-b) / 2$. The symbol $Q_{1}{ }^{0}$ is the associated Legendre function of the second kind. The cloud can be contained by a harmonic well, in which the potential varies as

$$
V(r, z)=V_{T}\left(2 z^{2}-r^{2}\right)\left(2 z_{0}^{2}\right)
$$

with voltages $V_{T}$ on the end electrodes consistent with the z-dependence of equation 3 . The harmonic well defines a natural axial frequency $\omega_{z}=\operatorname{sqrt}\left(\mathrm{qV}_{\mathrm{T}} /\left(2 \mathrm{mz}_{0}\right)^{2}\right)$, which is related to the stability of the cloud. With the HiPAT geometry the spheroidal cloud shape parameters $r_{0}$ and $z_{0}$ are approximately 2.7 and $11.7 \mathrm{~cm}$, respectively. Solving for $V_{T}$ with varying $n$ (similar approach as with magnetic field calculation) results in the curve shown in Figure 2 . From this data it can be seen that approximately $20 \mathrm{kV}$ and $10 \mathrm{kG}$ are required to hold the program goal of $10^{12}$ antiprotons.

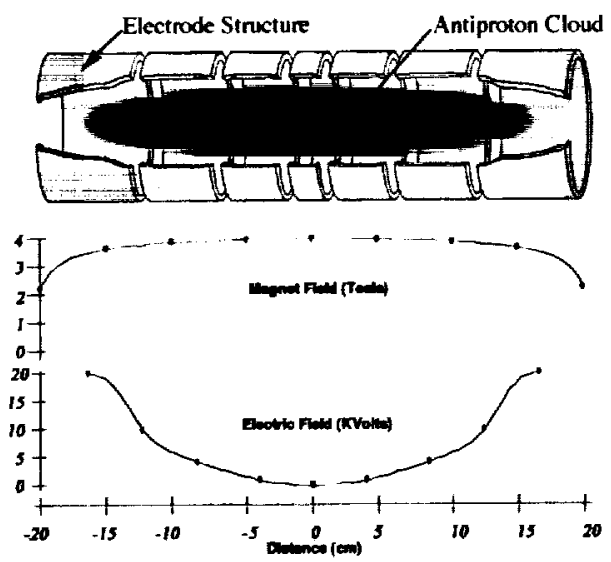

FIGURE 1. Electrode Layout.

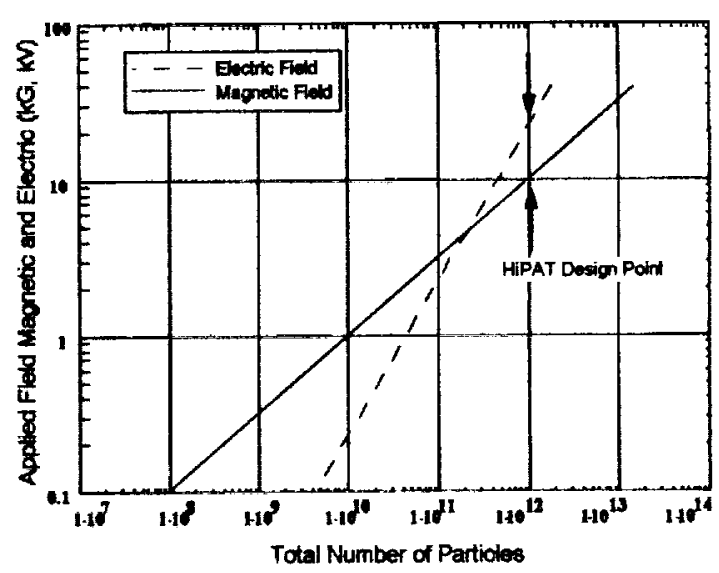

FIGURE 2. Trapped Particles vs. Applied Fields.

\section{Radial Diffusion and Other Loss Mechanisms}

There are several mechanisms that result in a loss of particles from the trapping volume. These loss rates are important since they dictate the extent of portability. The primary driver to loss or rather particle "lifetime" is the quality of the vacuum level which drives the radial diffusion and annihilation rates for trapped antiprotons. For a trap in which radial diffusion is dominated by background gas scattering, the mean lifetime (Bollen, 1996) can be expressed as

$$
\tau_{\mathrm{dif}}=1 / \mathrm{nov} *\left(\omega_{\mathrm{d}} / \omega_{2}\right)^{2}
$$

with $\sigma$ the momentum-transfer cross section, $v$ the relative velocity of the ions and background gas. The factor $\left(\omega_{\mathrm{c}} / \omega_{z}\right)^{2}$ arises from the random walk nature of the diffusion process, in which a scattering event is more likely to increase than to decrease the radial location of an ion. One feature predicted by equation 5 , is that lifetime increases as the square of magnetic field. The sensitivity of lifetime to magnetic field has been reported (Malmberg, 1980) experimentally. The lifetime also showed an increase with a decrease in background gas pressure, down to a pressure of $10^{-3}$ torr. The lifetime did not improve with improved vacuum below this value, because of the effects of perturbations due to alignment errors. But even with the alignment errors, the lifetime increased quadratically with magnetic field. Subsequent development of the traps pioneered by (Malmberg, 1980) has included the development (Hollmann, 2000) of the "rotating wall", in which r.f. with a cyclic phase pattern is imposed on a segmented 
cylindrical electrode. With this r.f. manipulation, up to $10^{9}$ ions have been held for several weeks. This technique will be utilized on HiPAT to provide a comfortable margin for portability.

Experimental tests of antiproton storage present challenges to theoretical models. In (Holzscheiter, 1996) the vacuum was poor enough (10 $10^{-11}$ torr range) to allow significant $\bar{p}$-atom formation. To illustrate extreme examples of theoretical uncertainties, consider the annihilation rates of $10 \mathrm{eV} \bar{p}$ 's in a $10^{-12}$ torr vacuum at $300 \mathrm{k}$. In one model, wherein direct annihilations are emphasized (Gibbs, 1997), the cross section in a helium background is 50 kbarns, implying a lifetime of 4 years. In another model, in which atom formation is taken as the basic picture (Morgan, 1970), the cross section in a hydrogen background is on the order of gigabarns, implying a lifetime of 5 hours. Despite the large differences, both models predict that above the tens of $\mathrm{eV}$ range the cross section drops rapidly. Above this energy $\bar{p}$-atom formation becomes improbable, and the cross sections approach the directannihilation limit. Fortunately for HiPAT the $\bar{p}$ 's require large energies, up to tens of $\mathrm{keV}$, nudged by r.f. to form the cloud rotation pattern needed for high density storage. The r.f. technique will be included in initial tests of antiproton storage in HiPAT.

\section{TRAP HARDWARE DESCRIPTION}

The HiPAT consists of a super conductive magnet system, ultra high vacuum system, high voltage distribution network, and trap electrode assembly as illustrated in Figures 3 and 4. To gain a basic understanding of portability from the start, these systems are mounted on a movable frame structure. The super conductive magnet, manufactured by Cryomagnetics Inc., is cooled by a combination of liquid helium and nitrogen and equipped with a two stage cryogenic cooler. The magnet $(\mathrm{NbTi} / \mathrm{Cu})$ is an end compensated solenoid design capable of producing a field strength of 4 tesla (providing sufficient margin over the required $10 \mathrm{kG}$ ).

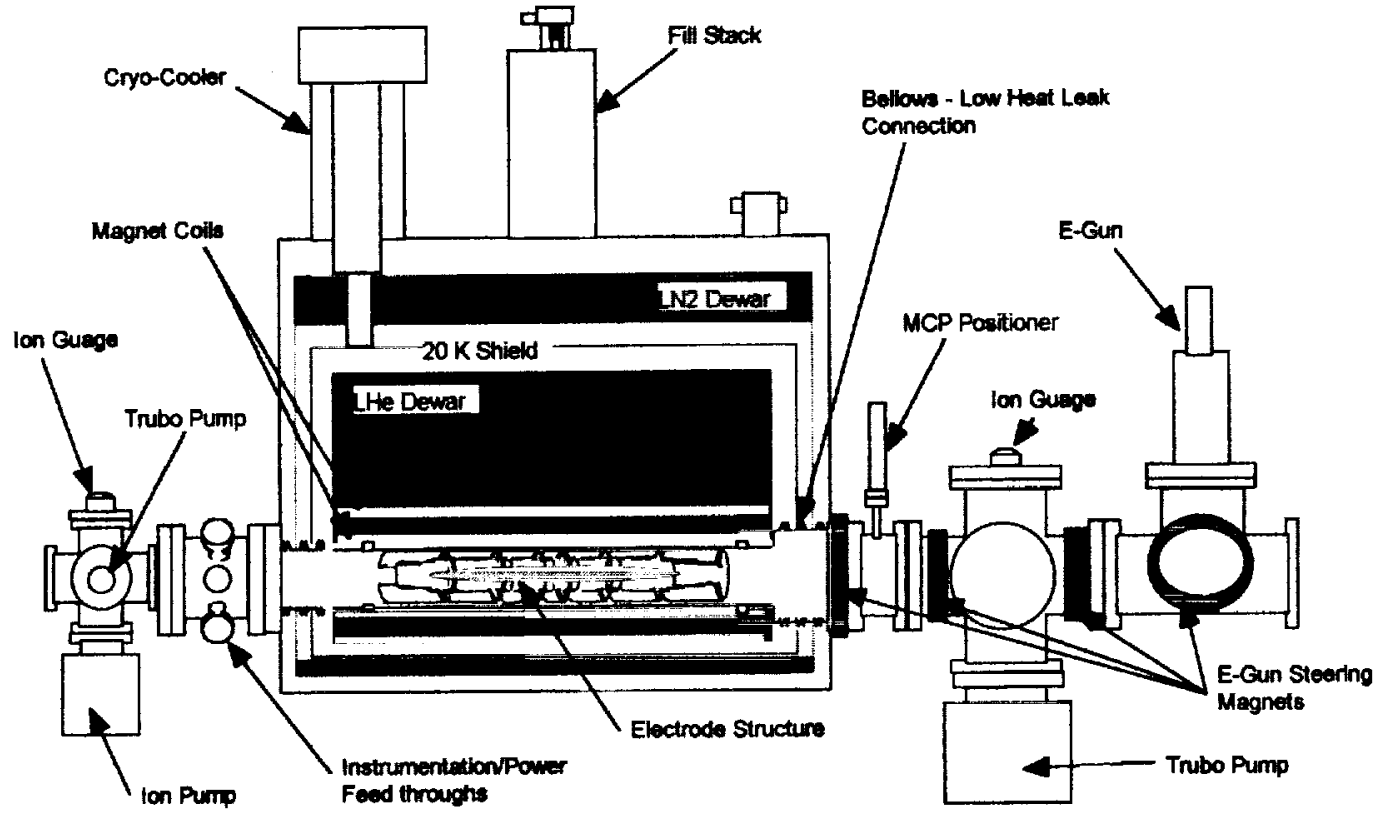

FIGURE 3. HiPAT Cross-Section.

It is also equipped with an internal active shield that effectively reduces the field to less than $50 \mathrm{G}$ at the boundary of the magnet system, simplifying operation and handling procedures. The system was designed around a $10 \mathrm{~cm}$ diameter $4 \mathrm{~K}$ central bore with the liquid helium tank exposed, providing cryopumping capability to the trapping region.

The ultra high vacuum system is dewar mounted and consists of a central region with integrated front and rear pumping manifolds. Primary pumping is performed with two turbo pumps and one ion pump which when operated 
with the cold bore are currently capable of reducing the system pressure to as low as $8.5 \times 10^{-11}$ torr as read on nude ion gauges. The vacuum system components were selected to allow for a minimum bake out temperature of $200 \mathrm{C}$. The forward manifold was kept clear of obstructions to allow for particle injection/extraction. It also contains a movable microchannel plate (MCP) device, which can be lowered into the beam line and used to make particle time of flight and quantity measurements. The rear vacuum manifold contains the main high voltage wiring and is equipped with feed-throughs capable of handling up to $20 \mathrm{kV}$. There are also allocations for providing RF input and signal output from the trap.

The trap assembly positioned in the magnet central bore, consists of a series of six electrodes centered on two pickup rings that are each composed of four 90 -degree sectors. The electrodes, manufactured from titanium, form a region $6 \mathrm{~cm}$ in diameter and $40 \mathrm{~cm}$ in length. To support and insulate the electrodes from each other and the beam line, an exoskeleton was fabricated from Macor, a machineable vacuum compatible glass product. Connections to all electrodes were made using $0.25 \mathrm{~mm}$ diameter kapton coated wire, which was routed though Alumina insulation tubes to the high voltage feed-throughs.

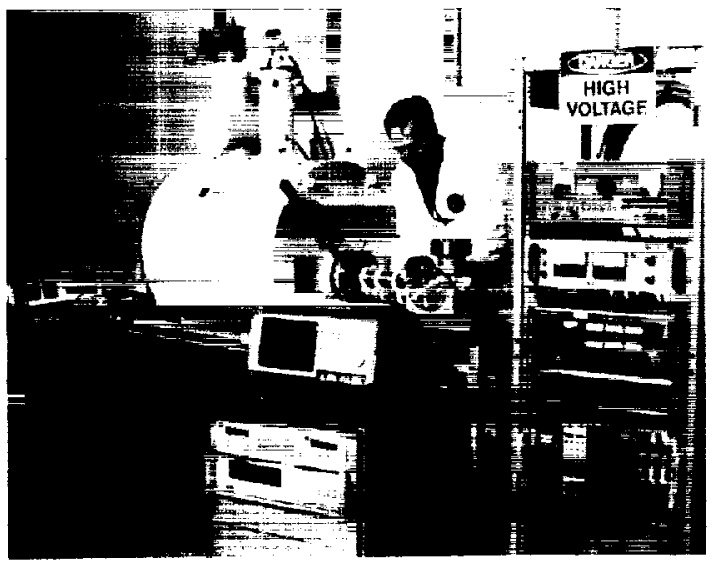

FIGURE 4. HiPAT Laboratory.

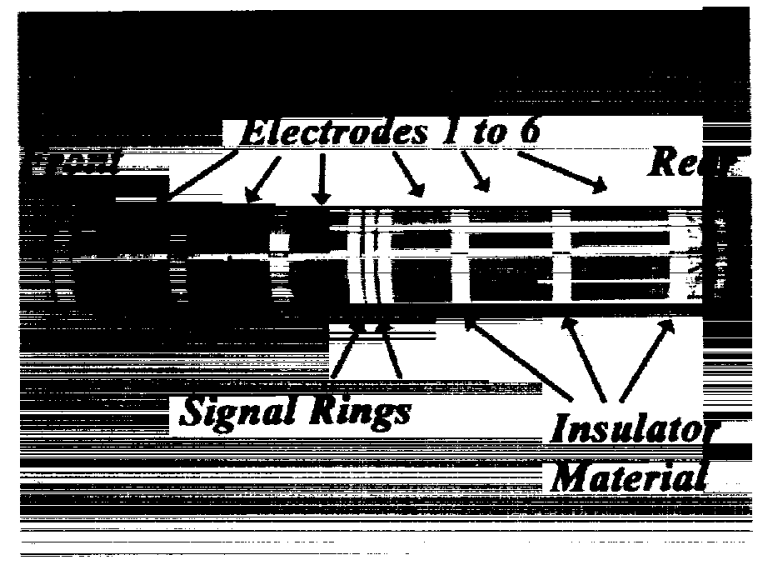

FIGURE 5. HiPAT Electrode Structure.

\section{CURRENT TESTING}

Testing to date has focused on storing positive ions to address both quantity and lifetime issues. These particles are generated within HiPAT using an electron gun to ionize background gas. Two methods were used to examine these trapped ions, the first, a non-intrusive technique made use of quadrupole pick ups to measure induced charge from ions within the cloud. This signal is then amplified and monitored with a spectrum analyzer. The second method requires destructively dumping the ions onto a MCP, providing time of flight and particle yield data. During initial testing, the induced signal technique could not be interpreted due to ambiguous data. The MCP however produced data more easily interpreted and so was chosen as the primary method of quantifying trap operation. The induced signal technique will be revisited once predictable behavior has been established.

During initial tests of this system it was found that a significant bluish glow discharge was produced within the negative well potentials formed at each end of the positively charge electrode structure. This discharge was visually observed to have the shape of a hollow column. The impact of this glow was three-fold, first it adversely impacted the vacuum pressure, second it resulted in unacceptable current draw from the electrode power supplies, and lastly it resulted in a condition where ions could be continuously supplied to the trapping region. The glow was also the cause of the numerous large ambiguous signals ranging from $100 \mathrm{kHz}$ to $5 \mathrm{MHz}$ measured by the induced signal technique. The discharge was investigated using optical spectral analysis, which indicated that the glow was the primary spectral emission for carbon monoxide (a contaminant in most ultra high vacuum systems). To combat the discharge several steps were taken, first the copper electrode material was replaced with titanium, second, floatable spoiler screens were added to both the front and rear of the trap to reduce the effect of negative wells, and lastly the system was periodically allowed to clean itself overnight by sustained glow discharge at high voltage $(15,000$ volts) and current ( 10 to 100 's of micro-amps). Typical trap operation is then restricted to below 1000 volts to provide a stand off against glow discharge. 
During initial proton storage tests, the effects of chemistry on the lifetime of the hydrogen ions were dramatic. A particular case illustrated in Figure 6, shows the results of a storage test using the forward portion of the trap (front three electrodes) to create a shallow elevated well with potentials of 1000,950 and 1000 volts above ground. The magnetic field was set to $20 \mathrm{kG}$ and the vacuum pressure maintained at $2 \times 10^{-10}$ torr. The electron gun was fired for 2 seconds with a maximum beam current of 50 micro-amps resulting in a temporary vacuum level of $7 \times 10^{-10}$ torr. Trap dumps to the MCP at four time slices are shown. The 3 second frame shows the initial constituents produced, primarily the hydrogen ion sequence $\mathrm{H}^{+}, \mathrm{H}_{2}^{+}$and $\mathrm{H}^{+}$with a slight amount of $\mathrm{CO}^{+}$. The time evolution shows the charge exchange between the hydrogen and the carbon monoxide as they are reduced and increased, respectively. The lifetime of hydrogen ions under these condition is very short, driven by chemistry, requiring that the quality of the vacuum be greatly improved (additional conditioning to remove carbon compounds). For each time panel in Figure 6 approximately 20,000 total ions are represented. Subsequent tests after additional trap conditioning resulted in increased lifetimes of the hydrogen ions and lower production rates of carbon monoxide.

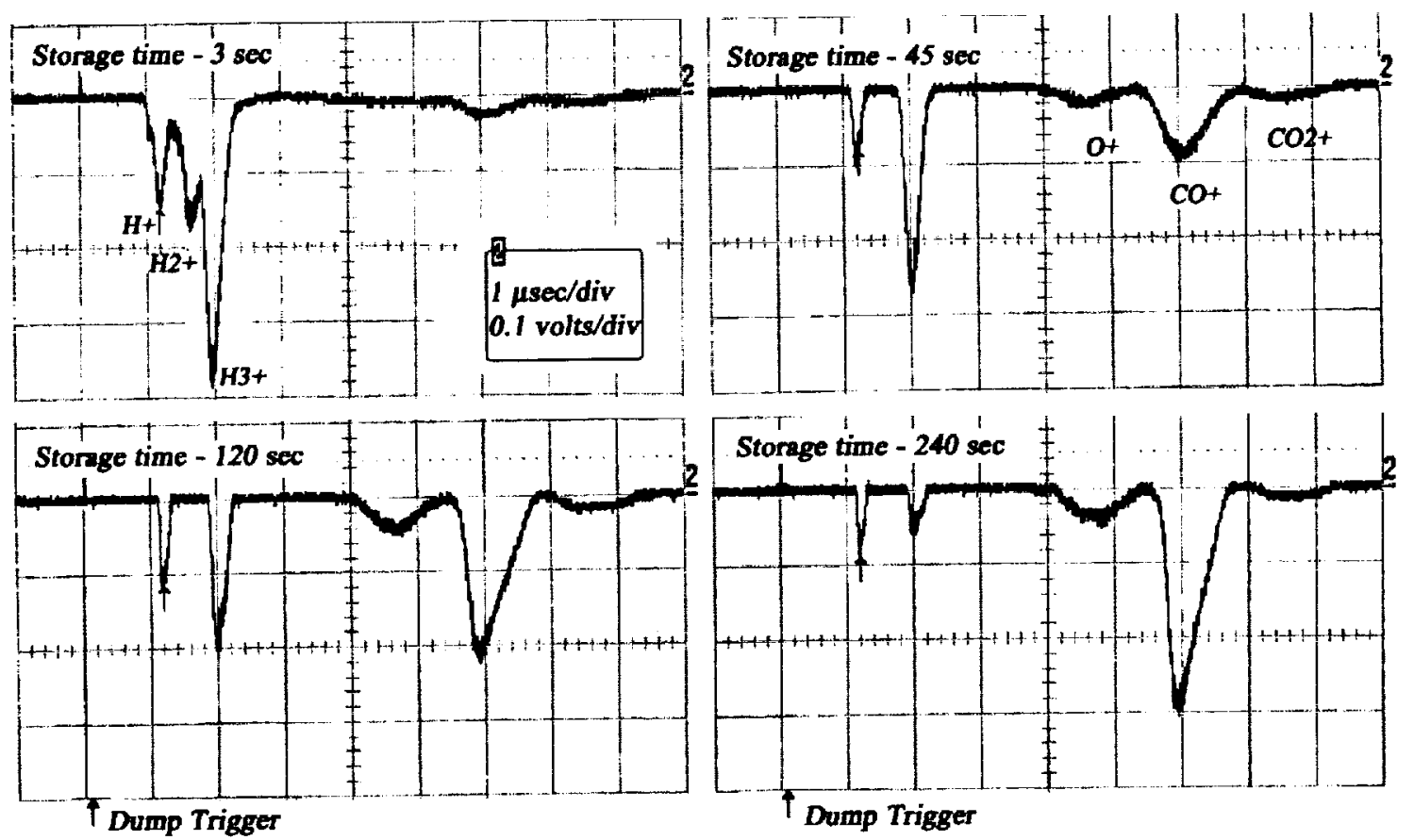

FIGURE 6. Ion Storage and Extraction From HiPAT Without R.F.

With the initial success of proton storage in HiPAT the application of a rotating wall r.f. pattern to the 4 segment ring (central) electrodes at the cyclotron frequency of $\mathrm{H}^{+}$ions was investigated. Using loading and vacuum conditions similar to those of the non r.f. test (described above), the trap potential well was set using all electrodes with a symmetric $500,300,200$ volt pattern and the center pickups floated at 100 volts. The magnetic field was adjusted to $19.7 \mathrm{kG}$ with a corresponding r.f. output of $30.37 \mathrm{MHz}$ (sum of magnetron and cyclotron frequencies (Gabrielse, 1990)) at $100 \mathrm{mV}$ amplitude. Figure 7 illustrates the dramatic effect of r.f. on the ion cloud with time panels ranging from 15 to 600 seconds. The double curves, shown on each panel in this figure, represent the same time-of-flight data but with different vertical scales to resolve both large and small features. After a few seconds, only $\mathrm{H}^{+}$ions are detected in the MCP, with merely hints of heavier ion clusters. Without the r.f. excitation, highmass ion clusters are formed at the expense of $\mathrm{H}^{+}$ions.

The r.f. technique is still unsatisfactory at present. Observations suggest that heating accompanying the compression process causes outgassing from the electrodes. The vacuum pressure is noted to drop when the trap is dumped (order of a few $\times 10^{-12}$ torr) implying that the ion cloud might be getting replenished from an extraneous mechanism. This condition is also hinted with the observation that the MCP baseline (r.f. on case) is not restored after the large hydrogen peak. The combination of r.f. frequency and amplitude have to be adjusted, to achieve stabilization with an acceptable amount of heating. Additionally, timed optical exposures of the trapping region will be required to verify that small pockets of glow discharge do not remain. 


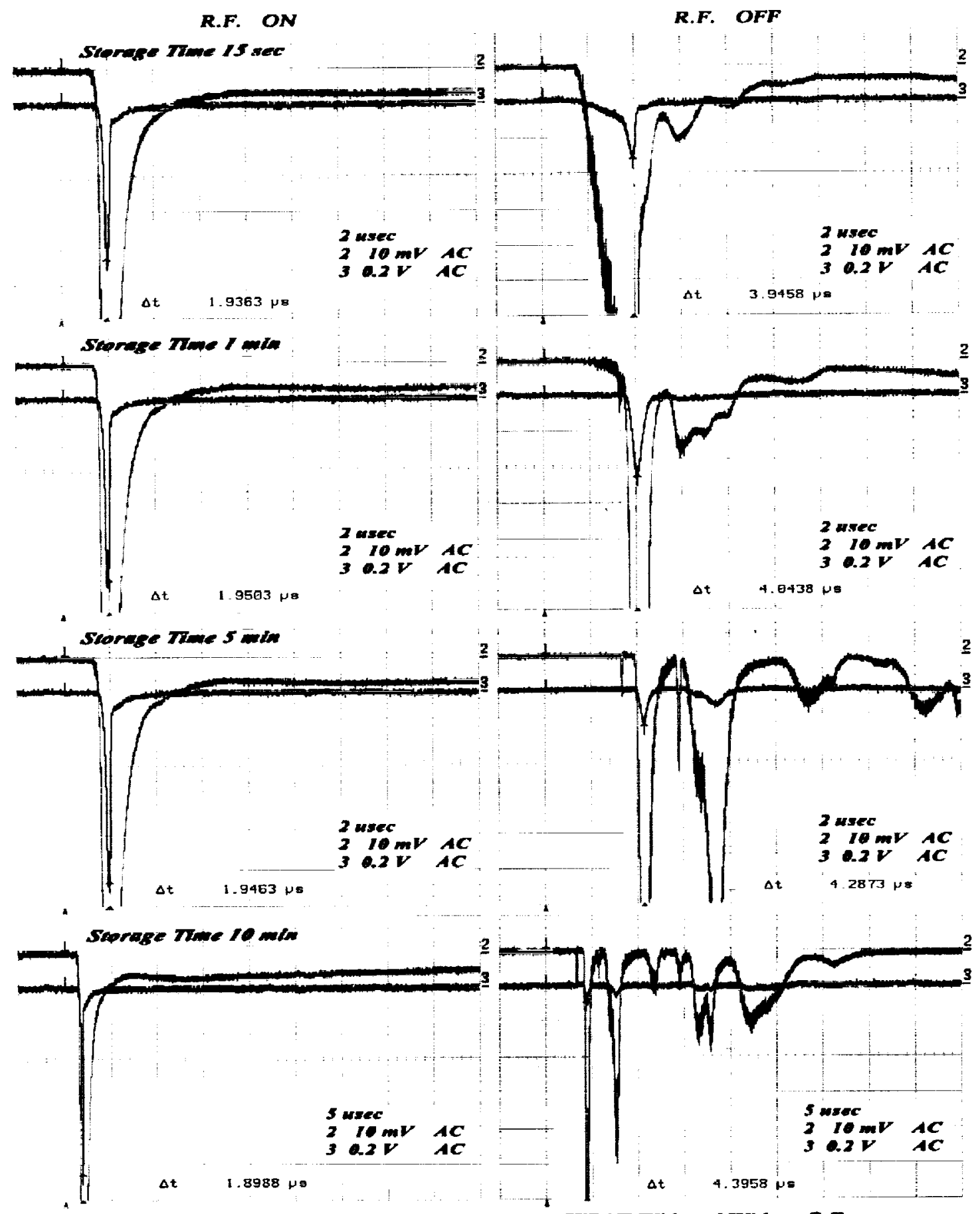

FIGURE 7, Ion Storage and Extraction From HiPAT With and Without R.F.

\section{FUTURE RESEARCH}

Improvements in the layout and conditioning of the ultra high vacuum system are scheduled. These changes are focused on improving the quality of vacuum (lowering the carbon based contaminants) as well as driving the ultimate pressure to the low $10^{-11}$ torr range. Modification of the electrode structure to improve the performance of the spoiler screens and better manage the potential for glow discharge is also planned. This effort is deemed critical since any hint of discharge makes the test data suspect. Once r.f. behavior with positive ions is better understood 
and stable extended ion storage achieved, negative well potentials using electrons and negative hydrogen ions will be investigated. Control of the discharge phenomena is critical to these tests since the negative ions and potential glow discharge occupy the same containment volume. Also, positive and negative hydrogen ion sources have been procured. These sources will provide the capability of practicing dynamic capture and stacking, techniques required for filling the trap with antiprotons.

\section{ACKNOWLEDGMENTS}

The authors wish to acknowledge the contributions to this project made by George Schmidt, Harold Gerrish, Leo Bitteker, Chris Dobson, Gene Fant and Kevin Pedersen.

\section{REFERENCES}

Augenstein, B.W., "RAND Workshop on Antiproton Science and Technology," RAND Note $\mathrm{N}-2763-\mathrm{AF}$, October 1988

Bollen G. et. al., "ISOLTRAP: a Tandem Penning Trap System for Accurate On-line Mass Determination of Short-lived Isotopes," Nucl. Inst \& Meth. In Phys. Res. A 368, 675, 1996

Bollinger, J.J., et. al., "Electrostatic Modes of Ion-Trap Plasma," Phys. Rev. A 48, 525, 1993

Borowski, S.K., "A Comparison of Fusion/Antiproton Propulsion Systems For Interplanetary Travel," AIAA-87-1814, Joint Propulsion Conference, 1987

Brillouin, L., "A Theorem of Larmor and Its Importance for Electrons in Magnetic Fields," Phys. Rev., Vol. 67, No. 7, 260, 1945

Frisbee, R.H., and Leifer, S.D., "Evaluation of Propulsion Options for Interstellar Missions," AIAA-98-3403, Joint Propulsion Conference, July 1998

Gabrielse, G., et. al., "Thousandfold Improvement in the Measured Antiproton Mass," Phys. Rev. Let., $65,1317,1990$

Gaidos, G., et al., "Antiproton-Catalyzed Microfission/Fusion Propulsion Systems for Exploration of the Outer Solar System and Beyond," AIAA 98-3589, Joint Propulsion Conference, July 1998

Gaidos, G., et al., "AIMStar: Antimatter Initiated Microfusion for Re-cursor Interstellar Missions," AIAA-98-3404, Joint Propulsion Conference, July 1998

Gibbs, W.R., "Low-energy Antiproton Interaction with Helium," Phys. Rev. A, Vol. 56, No. 5, 3553, 1997

Hollmann, E.M., et. al., "Confinement and Manipulation of Non-Neutral Plasmas Using Rotating Wall Electric Fields," Phys of Plasmas, Vol. 7, No. 7, 2776, 2000

Holzscheiter, M.H., et. al., "Are Antiprotons Forever?," Phys. Let. A, Vol. 214, pp 279-284, 1996

Howe, S.D., "Portable Pbars, Traps that Travel", Los Alamos National Laboratory Report, LA-UR 88-737, 1988

Kammash T., and Galbraith, D.L., "Antimatter-Driven Fusion Propulsion Scheme for Solar System Exploration," Journal of Propulsion and Power, Vol. 8, No. 3, May 1992

Malmberg, J.H., "Long-Time Containment of a Pure Electron Plasma," Phys. Rev. Let., Vol. 44, No. 10, $654,1980$.

Morgan., D.L., et. al., "Atomic Processes Involved in Matter-Antimatter Annihilation," Phys. Rev. D, Vol. 2, No. 8, 1389, 1970

Morgan, D.L., "Concepts for the Design of an Antimatter Annihilation Rocket," J. British Interplanetary Society, Vol 35.9, 1982

Perkins, L.J., "Antiproton Fast Ignition for Inertial Confinement Fusion," Fusion Technology, Vol. 36, pp 219-233, Sept 1999

Rider, T.H., "Fundamental Constraints on Large-Scale Antimatter Rocket Propulsion, " Journal of Propulsion and Power, Vol. 13, Nov 3, 1997

Schmidt, G.R., et. al., "Antimatter Production and Energy Costs for Near-Term Propulsion Applications," Journal of Propulsion and Power, Vol.16, No. 5, 923-928, 2000

Smith, G.A. et. al., NASA JPL Penn State University Contract 960744 Titled, "Antiproton -Catalyzed Microfission/Fusion Propulsion", 1997

Vulpetti, G. "A Propulsion-Oriented Synthesis of the Antiproton-Nucleon Annihilation Experimental Results", J. British Interplanetary Society, 37 (1984), pp. 124-134. 